\title{
An Ultrawideband Microfabricated Gold-Based Antenna Array for Terahertz Communication
}

\author{
Abdoalbaset Abohmra ${ }^{\circledR}$, Student Member, IEEE, Hasan Abbas ${ }^{\circledR}$, Member, IEEE, \\ Jalil ur Rehman Kazim ${ }^{\circledR}$, Student Member, IEEE, Muhammad S. Rabbani ${ }^{\circledR}$, Member, IEEE, \\ Chong Li ${ }^{\circledR}$, Senior Member, IEEE, Akram Alomainy ${ }^{\circledR}$, Senior Member, IEEE, \\ Muhammad A. Imran ${ }^{\circledR}$, Senior Member, IEEE, and Qammer H. Abbasi ${ }^{\circledR}$, Senior Member, IEEE
}

\begin{abstract}
The microwave frequency band typically used for wireless communications will soon become saturated and will no longer be able to fulfill the high bandwidth demands of modern communication networks. Terahertz $(\mathrm{THz})$ communication has appeared as a highly attractive, future-generation wireless technology that offers higher spectral bandwidth and, therefore, higher data rates. However, the full exploitation of $\mathrm{THz}$ technologies is contingent upon the availability of energy-efficient sources and devices. In this letter, we present a fabrication and measurement of a microscale planar inverted cone antenna array made of gold. Using an ungrounded coplanar waveguide (CPW) feed, the microfabricated structure provides a bandwidth of $37.9 \%$ with the resonant frequency of $0.925 \mathrm{THz}$. Given the cost of microfabrication is reduced substantially with rapid technological advancements, the results of this letter suggest that high-speed $\mathrm{THz}$ communications can be realized for wide-scale applications.
\end{abstract}

Index Terms-Antenna, gold, planar inverted cone antenna (PICA), terahertz (THz).

\section{INTRODUCTION}

$\mathbf{T}$ HE global average monthly mobile data consumption per device is expected to rise to $24 \mathrm{~GB}$ in 2025 [1], which will lead to great strains on the mobile networks. Terahertz (THz) communications have naturally been tagged as an attractive solution to the expected bandwidth crunch as $\mathrm{THz}$ networks have a potential capacity in the range of several terabits per second [2]-[4]. However, the device technology to build sources and detectors is still in its infancy as compared to the established microwave and optical counterparts, which cannot be merely scaled

Manuscript received March 10, 2021; revised April 4, 2021; accepted April 7, 2021. Date of publication April 12, 2021; date of current version November 16, 2021. This work was supported by the Engineering and Physical Sciences Research Council (EPSRC) under Grant EP/R511705/1. (Corresponding author: Abdoalbaset Abohmra.)

Abdoalbaset Abohmra and Jalil ur Rehman Kazim are with the James Watt School of Engineering, University of Glasgow, G12 8QQ Glasgow, U.K (e-mail: 2356006a@student.gla.ac.uk; j.kazim.1@ research.gla.ac.uk).

Hasan Abbas, Chong Li, Muhammad A. Imran, and Qammer H. Abbasi are with the James Watt School of Engineering, University of Glasgow, E1 4NS London, U.K. (e-mail: Hasan.Abbas@glasgow.ac.uk; Chong.Li@glasgow.ac.uk; Muhammad.Imran@glasgow.ac.uk; Qammer.Abbasi@glasgow.ac.uk).

Muhammad S. Rabbani is with the School of Electronic, Electrical and System Engineering, University of Birmingham, B15 2TT Edgbaston, U.K. (e-mail: m.s.rabbani@bham.ac.uk).

Akram Alomainy is with the School of Electronic Engineering and Computer Science, Queen Mary University of London, E1 4NS London, U.K. (e-mail: A.alomainy@qmul.ac.uk).

Digital Object Identifier 10.1109/LAWP.2021.3072562 to $\mathrm{THz}$ frequencies owing to many reasons. $\mathrm{THz}$ technologies have been translated into various applications that span biomedical science and engineering [5], [6], imaging schemes [7], [8], military [9], calibration systems for microwave sensors [10], space instrumentation [11], and environmental monitoring systems [12]. Most of the aforementioned applications particularly exploit the unique interactions of water molecules with the $\mathrm{THz}$ waves [13]. The so-called $\mathrm{THz}$ gap ranges from 0.1 to $10 \mathrm{THz}$ and this frequency range is less affected by adverse climatic conditions such as rain and fog [14]. However, with respect to the realization of $\mathrm{THz}$ wireless systems, it remains a challenge to realize an ultrawideband (UWB), low-cost antenna that is scalable and easily integrated on-chip [15]. In this regard, a variety of $\mathrm{THz}$ nanoantenna designs that are scaled versions of the microwave frequency counterparts have been recently proposed that include printed dipoles [16]-[18], and simple patch antennas [19]. On-chip THz beam-forming array systems that are fabricated on silicon have been also reported [20], [21]. Surface plasmon enabled enhancement of the near-field further extends the promise of dipole-type antennas for $\mathrm{THz}$ communications [17]. Similarly, a nanoantenna array was also developed for subwavelength focusing of the THz waves [19]. A theoretical analysis of a highly directed antenna array having 16 elements at $0.3 \mathrm{THz}$ was shown in [20] with a maximum directivity of $18.1 \mathrm{dBi}$. Despite these various attractive antenna designs, the patch antenna has the advantage of low cost and relatively simple fabrication achieved through conventional semiconductor deposition techniques [15]. At THz frequencies, the substrate thickness inevitably becomes comparable to the wavelength [22], which prevents the efficient design of the planar antenna feeding networks, due to the change in the impedance. Typically, the substrate thickness is often set in the range of two orders of magnitude below the operating frequency, so that radiation efficiency of the antenna can be improved. However, at $\mathrm{THz}$ frequencies, such a procedure results in extremely thin substrates, which adversely affect the structural integrity of the antenna. A common practice is to place a dielectric lens, typically made of silicon below the substrate [23], which is inspired by the use of $\mathrm{THz}$ technology in astrophysics. However, for applications in which planar designs are preferred, such a remedial measure to enhance the substrate radiation becomes unusable. Other techniques involve the use of thin films of dielectric membranes embedded within waveguides [24]. 
Due to the complicated designs, such measures have not been broadly applied. The ungrounded coplanar waveguide (CPW) feed is a viable solution to provide not only just good impedance matching with a sufficient substrate thickness, but low transmission loss, small frequency dispersion, and most importantly easy integration with the monolithic circuit designs [25]. However, the size and complicated structure of the antenna becomes a big concern at the $\mathrm{THz}$ band. The limitations of material and fabrication capability at this small size limited the consideration of new antenna geometry with new techniques. A corporate feed antenna array with slot radiators and a layer of polarizing patches is designed to operate at $350 \mathrm{GHz}$ [26]. An UWB antenna covering the lower THz band is designed and numerically analysed [27]. However, the size and structural complexities of the device become a great issue at the $\mathrm{THz}$ frequency. A $3.2 \mathrm{THz}$ array of patch antenna microcavities connected by narrow plasmonic wires presented in [28] demonstrates THz lasers made of arrays of $10 \times 10$ patch antenna microcavities. With CPW feeding techniques, a wider bandwidth can be obtained. The CPW PICA antenna as compared to other techniques [29]-[31] has a better gain and efficiency [32]. In addition, using GSG CPW antenna measurement simplifies the structure and makes the antenna easy to fabricate, which makes the antenna cost-effective. Among the antenna designs that yield an UWB response with an omnidirectional spatial coverage, the planar inverted cone antenna (PICA) has emerged as one of the most promising designs in the last two decades. A PICA is composed of a semicircle, which is extended into an inverted cone from the flat side. Performance-wise, the PICA is known to produce an impedance bandwidth of 20:1 at microwave frequencies [33], [34]. Moreover, due to the flat profile, the PICA exhibits mechanical stability due to which it is increasingly being used for commercial applications that require high bandwidth in an omnidirectional way. In this letter, we presented for the first time in the literature, a fabricated and experimentally measured, novel and simple UWB omnidirectional $1 \times 4 \mathrm{THz}$ PICA array with a $37.9 \%$ bandwidth with a central frequency of $0.92 \mathrm{THz}$. The simple profile of the antenna array and excellent radiation characteristics can pave the way for realizing truly UWB wireless communications in the $\mathrm{THz}$ frequency band.

\section{A. Antenna Design and Fabrication}

The proposed antenna design was simulated and analyzed using a three-dimensional, full-wave commercial electromagnetics solver, Dassault Systemes CST Studio Suite 2020. The dimensions of the antenna and the feeding lines are described in Table I and the designed antenna structure along with the feeding network is shown in Fig. 1. An array of gold-based PICAs was designed at $1 \mathrm{THz}$ on a silicon substrate, having a dielectric constant, $\varepsilon_{r}=11.9$ and thickness, $h=600 \mu \mathrm{m}$. The initial dimensions of the PICA were obtained using standard microstrip patch antenna design equations, with the help of which we calculated the width and length of an equivalent patch antenna resonating at $1.00 \mathrm{THz}$. Following the design guidelines [35], an inverted cone was generated where the dimensions $W_{p}$ and $L_{p}$ were calculated using the expressions provided in our previous work [36]. In the PICA array, the antenna element spacing was set to a $\lambda$ at $1 \mathrm{THz}$.
TABLE I

DIMENSIONS OF THE GOLD PICA ARRAY

\begin{tabular}{|l|l|l|}
\hline Dimensions & Description & Value $(\mu \mathrm{m})$ \\
\hline$g$ & CPW feeding gap & 1 \\
\hline$L_{p}$ & Patch Length & 100 \\
\hline$W_{p}$ & Patch width & 120 \\
\hline$W_{s}$ & Substrate width & 1000 \\
\hline$L_{s}$ & Substrate length & 2000 \\
\hline$h$ & Substrate thickness & 600 \\
\hline$W_{f}^{50}$ & $50 \Omega$ feed line width & 45 \\
\hline$W_{f}^{70}$ & $70 \Omega$ feed line width & 20 \\
\hline$W_{f}^{100}$ & $100 \Omega$ feed line width & 9.9 \\
\hline$d$ & Element Spacing & 300 \\
\hline$L_{g}$ & Ground plane length & 978 \\
\hline
\end{tabular}

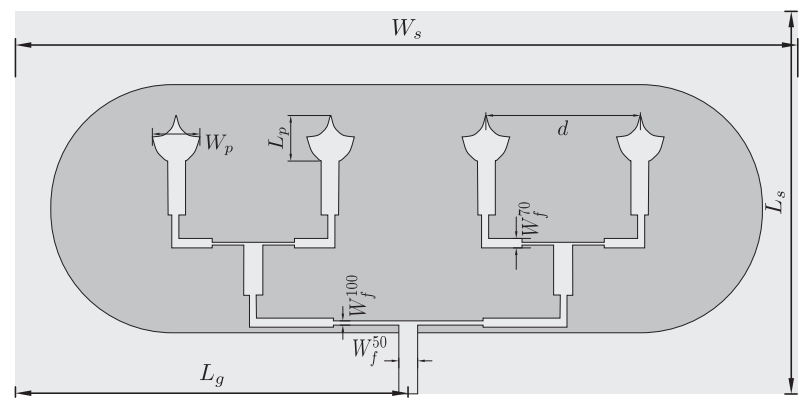

Fig. 1. Geometry and dimensions the PICA array and the feeding network.

The resultant design was then optimized using the trust-region framework (TSF) algorithm [37] included in CST. The TSF algorithm searches locally for minimal points in a given region. However, based on the parameters provided to the framework, the algorithm can operate locally as well. To find obtain the optimal antenna structure, a multidimensional problem in which all the physical dimensions of the PICA array were parametrized was rigorously solved using high-performance computing facilities at the University of Glasgow. The goal of the algorithm was to find the local minima for the initial values, which were calculated using standard microstrip patch antenna designs [38]. The fabrication process of the proposed $1 \times 4$ PICA array was carried out at the James Watt Nanofabrication Centre, University of Glasgow. To make the antenna resistant to corrosion and chemically stable, we chose gold as the radiating material. Besides, at $\mathrm{THz}$ frequencies, gold exhibits a high electrical conductivity with a lower skin depth ( $\sim 80 \mathrm{~nm}$ at $1 \mathrm{THz})$ [39]. It is easily deposited using traditional deposition techniques such as sputtering, evaporation, and electroplating with a high-melting point [40]. For the fabrication of submicron structures, electron beam lithography (EBL) is utilized to transfer the pattern onto a resist layer that is precoated on the surface of a wafer. In general, a shorter wavelength is the key to achieve smaller feature sizes. With the EBL nanoscale, THz antenna designs can be realized. The fabrication is carried out using a four-step process where the sample undergoes cleaning, after which the photoresist is spun on the wafer. After that, the design is transferred using the EBL and the last step involves the metal liftoff. The execution of these steps initially involved a polymethyl methacrylate (PMMA) resist, which was spin-coated onto the silicon substrate with a thickness of $600 \mu \mathrm{m}\left(\varepsilon_{r}=11.5, \tan \delta=0.0005\right)$ [41]. The 


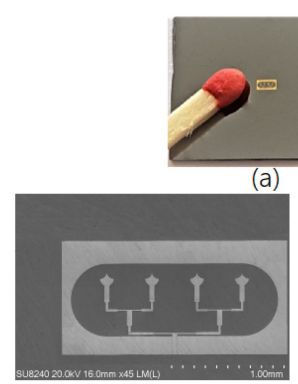

(b)

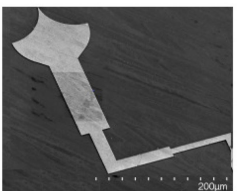

(c)
Fig. 2. (a) Comparison of the size of the fabricated $1 \times 4$ PICA array with a matchstick. (b) Array structure. (c) Feeding transmission line for the PICA array.

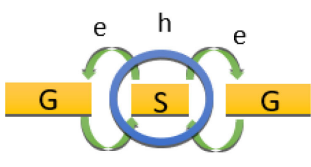

(a)

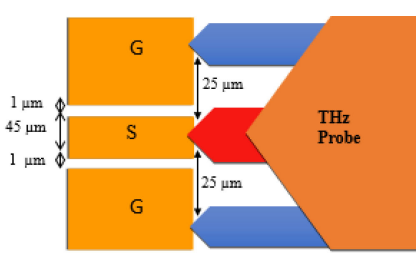

(b)
Fig. 3. Ungrounded CPW feeding network. (a) Illustration of the electric and magnetic field distributions around the ungrounded CPW and (b) schematic of the probe measurement contact with the central signal transmission line (S) and ground plane $(\mathrm{G})$.

higher sensitivity photo-resist requires a lower dose, which ultimately determines the exposure times. The exposure dose from the e-beam source was tested on the PMMA photoresist before the process of fabrication and it was found that $650 \mu \mathrm{m} / \mathrm{cm}^{2}$ dose was optimum to obtain a good resolution. A 2.5:1 developer was used to develop the PMMA for $30 \mathrm{~s}$ and rinsed afterward with isopropyl alcohol for $20 \mathrm{~s}$. Lastly, a $500 \mathrm{~nm}$ layer of gold was deposited on the developed wafer. The unwanted gold was removed by dissolving the resist in acetone solution for $4 \mathrm{~h}$. The size and geometry of the obtained fabricated structure can be ascertained from Fig. 2, which also contains images recorded from an ultrahigh-resolution field emission scanning electron microscope, SU8240 by Hitachi high-technologies.

\section{B. Measurement and Result}

As per the authors' knowledge, the fabricated on-chip antenna was measured in the $\mathrm{THz}$ frequency band, for the first time in the literature, where we used a Cascade Microtech $\mathrm{THz}$ wave vector network analyzer (VNA) coupled with a Virginia Diodes Inc. VNA extender to obtain the scattering properties of the PICA array in 0.75-1.10 THz frequency range. The VNA was connected with a separate set of submillimetre scale $\mathrm{THz}$ wave probes that provided an on-wafer ground-signal-ground $(\mathrm{G}-\mathrm{S}-\mathrm{G})$ electrical contact for the measurement in the desired frequency range of $0.75-1.10 \mathrm{THz}$. The $\mathrm{G}-\mathrm{S}-\mathrm{G}$ probe having a pitch of $25 \mu \mathrm{m}$ was carefully placed on the ungrounded CPW feeding line of the proposed antenna for measurement. Fig. 3(a) shows the electric and magnetic field distribution around the CPW line, whereas Fig. 3(b) illustrates how the THz probe was used to excite the ungrounded CPW feedline.

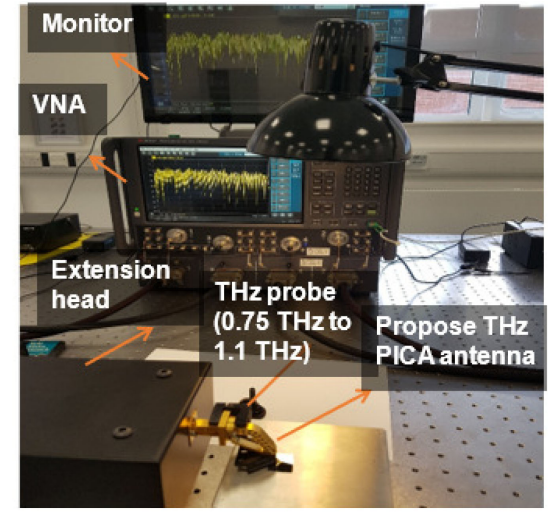

(a)
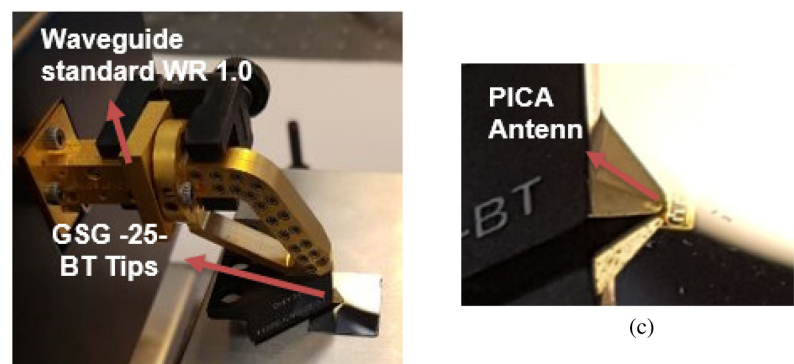

(c)

(b)

Fig. 4. Experimental setup used to measure the scattering parameters. (a) Eye view of the setup, (b) GSG-25-BT THz probe (0.75-1.10 THz) contact location and, and (c) placement of the probe on the PICA

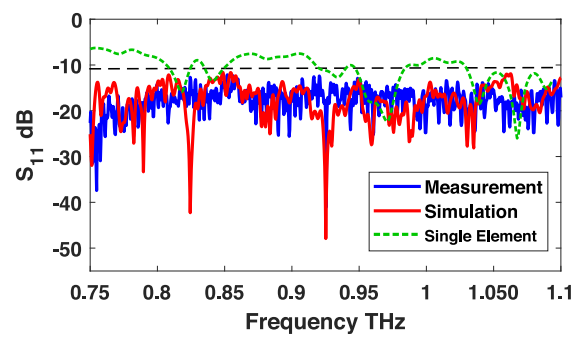

Fig. 5. Measured and simulated reflection coefficient of the $1 \times 4$ PICA array.

The complete measurement setup is illustrated in Fig. 4, which was configured at the Metamaterials Engineering Laboratory, University of Birmingham. The $\mathrm{THz}$ probe used offers a low insertion loss, i.e., $<1.5 \mathrm{~dB}$ with good sample visibility. The VNA was calibrated for 1-port $S$-parameter measurement using the short-open-load-through (SOLT) calibration process on a cascade impedance substrate standard. The simulated reflection coefficients of the single element and the four-element array as well the measurement results of the PICA array are shown in Fig. 5, which shows the excellent antenna performance as the whole response is below the $10 \mathrm{~dB}$ range. The difference between the single antenna and array response is attributed to the mutual coupling among the elements of the array. Moreover, both the measurement and simulation results exhibit a good agreement with each other, and the disparity is attributed to the unpredictable reflection from the surroundings of the experimental setup. In addition, the antenna was fabricated on a carrier wafer, which was kept larger than the simulated substrate to 


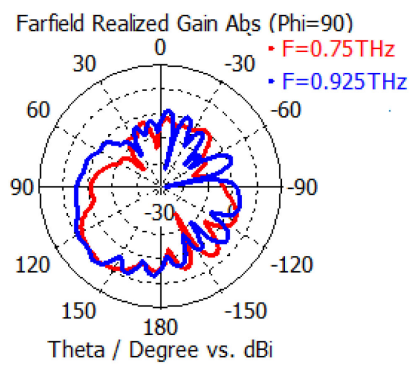

(a)

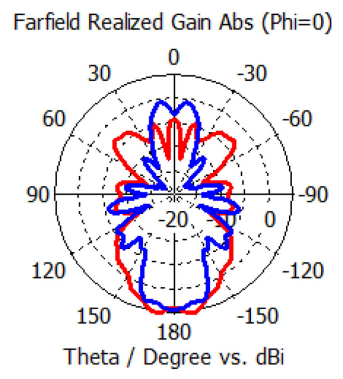

(b)
Fig. 6. Simulated radiation pattern of the proposed antenna array at two frequencies (a) 0.75 and (b) $0.925 \mathrm{THz}$.

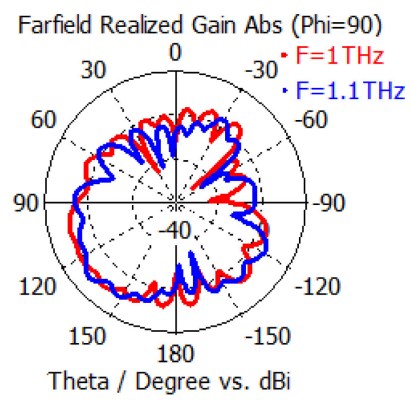

(a)

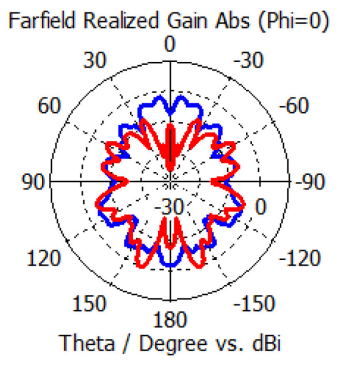

(b)
Fig. 7. Simulated radiation pattern of the proposed antenna array at different frequencies throughout the operating band. (a) $f=1.0 \mathrm{THz}$. (b) $f=1.10 \mathrm{THz}$.

ensure convenient handling of the fabricated antenna, resulting in a slight impedance mismatch with the feeding network. This can be observed by a series of ripples that can be seen in the measured scattering response. Figs. 6 and 7 present the simulated radiation patterns of the designed antenna array at $0.75,0.925,1$, and $1.10 \mathrm{THz}$, respectively. The radiation patterns are presented at E- and H-planes. Due to the large dielectric constant of the silicon substrate, most of the radiation is directed toward the substrate. This is a common problem with antennas fabricated on high dielectric constant substrates, which at $\mathrm{THz}$ frequencies is often circumvented by using a hemispherical lens. The sidelobe level is increased and the maximum side lobe is observed at $1.10 \mathrm{THz}$. Therefore, the ripples in the radiation pattern may lead to a degradation in the antenna performance, which is mainly caused by metal loss and skin depth effect. In Figs. 6 and 7 , the radiation patterns show high main lobe magnitudes along with lower back lobe levels. Fig. 8 shows the simulated antenna performance in terms of the antenna radiation efficiency and the gain achieved by the array in the operating frequency range. It can be seen that the gain of the antenna is maintained above $70 \%$. Although, this is lower as compared to antenna designs at microwave frequencies where the radiating element acts nearly as a perfect electric conductor, the conductivity of gold at $\mathrm{THz}$ frequencies is complex-valued where the imaginary part contributes to losses. Further investigations in which a substrate with a lower dielectric constant is used, can improve the radiation efficiency of the designed antenna. Other performance parameters such as the antenna gain and directivity are shown in Fig. 8(b) that indicates a high realized gain in the range of $12.5-16 \mathrm{dBi}$.

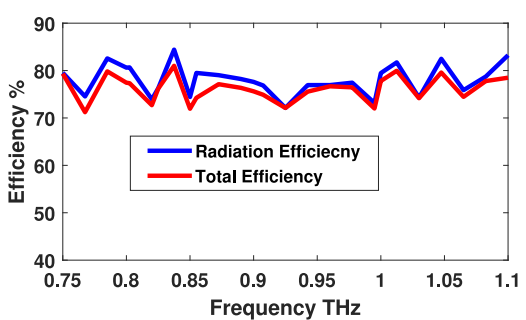

(a)

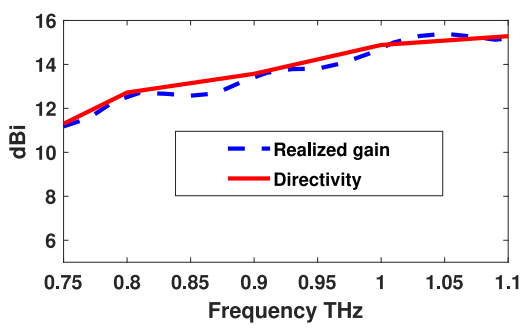

(b)

Fig. 8. Simulated results of the $1 \times 4$ PICA array (a) radiation and total efficiency and (b) realized gain.

\section{CONCLUSION}

In this letter, a gold-based $1 \times 4$ PICA array operating in the $\mathrm{THz}$ frequency range of $0.75-1.10 \mathrm{THz}$ is designed and fabricated. A simple and commonly available metal deposition technique to fabricate the structure is used. An ungrounded coplanar waveguide feeding line was used to excite the antenna. As per the authors' knowledge, the proposed design is the first instance of the $\mathrm{THz}$ antenna for which the scattering response was measured. Our design achieved a measured $-10 \mathrm{~dB}$ impedance bandwidth of $400 \mathrm{GHz}$ at the center frequency of $0.925 \mathrm{THz}$. Results also show that other antenna performance characteristics such as radiation efficiency and gain are also high. We anticipate the designs and techniques used in this letter can launch a new research direction for realizing $\mathrm{THz}$ frequency UWB antennas for enabling high-speed wireless communications in the future.

\section{ACKNOWLEDGMENT}

A. Abohmra would like to thank the Government of Libya for funding his doctoral studies.

\section{REFERENCES}

[1] Nidhi, A. Mihovska, and R. Prasad, "Spectrum sharing and dynamic spectrum management techniques in $5 \mathrm{G}$ and beyond networks: A survey," J. Mobile Multimedia, vol. 17, no. 1-3, pp. 65-78, 2021.

[2] M. Koch, "Terahertz communications: A 2020 vision," in Terahertz Frequency Detection and Identification of Materials and Objects. Berlin, Germany: Springer, 2007.

[3] J. Laskar, S. Pinel, D. Dawn, S. Sarkar, B. Perumana, and P. Sen, "The next wireless wave is a millimeter wave," Microw. J., vol. 50, no. 8, pp. 22-32, 2007.

[4] J. M. Jornet and I. F. Akyildiz, "Graphene-based plasmonic nano-antenna for terahertz band communication in nanonetworks," IEEE J. Sel. Areas Commun., vol. 31, no. 12, pp. 685-694, Dec. 2013.

[5] H. P. Siegel, "Terahertz technology in biology and medicine," IEEE Trans. Microw. Theory Techn., vol. 52, no. 10, pp. 2438-2447, Oct. 2004.

[6] A. J. Fitzgerald, E. Berry, N. N. Zinovev, G. C. Walker, M. A. Smith, and J. M. Chamberlain, "An introduction to medical imaging with coherent terahertz frequency radiation," Phys. Med. Biol., vol. 47, no. 7, 2002 Art. no. R67. 
[7] M. K. Choi, K. Taylor, A. Bettermann, and D. W. Van der Weide, "Broadband 10-300 GHz stimulus-response sensing for chemical and biological entities," Phys. Med. Biol., vol. 47, no. 21, 2002, Art. no. 3777.

[8] G.-J. Kim, W.-K. Han, J.-I. Kim, and S.-G. Jeon, "High resolution terahertz imaging (T-ray) with a horn antenna," in Proc. 35th Int. Conf. Infrared, Millimeter, Terahertz Waves, 2010, pp. 1-2.

[9] D. L. Woolard, J. O. Jensen, and R. J. Hwu, Terahertz Science and Technology for Military and Security Applications, vol. 46. Singapore: World Scientific, 2007.

[10] R. E. Cofield and P. C. Stek, "Design and field-of-view calibration of 114 $660-\mathrm{GHz}$ optics of the earth observing system microwave limb sounder,' IEEE Trans. Geosci. Remote Sens., vol. 44, no. 5, pp. 1166-1181, May 2006.

[11] H. P.Siegel, “THz instruments for space," IEEE Trans. Antennas Propag., vol. 55, no. 11, pp. 2957-2965, Nov. 2007.

[12] M. Tonouchi, "Cutting-edge terahertz technology," Nature Photon., vol. 1 , no. 2, pp. 97-105, 2007.

[13] D. L. Woolard, R. Brown, M. Pepper, and M. Kemp, “Terahertz frequency sensing and imaging: A time of reckoning future applications?" Proc. IEEE, vol. 93, no. 10, pp. 1722-1743, Oct. 2005.

[14] G. P. Williams, "Filling the $\mathrm{THz}$ gap-High power sources and applications," Rep. Prog. Phys., vol. 69, no. 2, p. 299-301, 2005.

[15] K.-M. Luk et al., "A microfabricated low-profile wideband antenna array for terahertz communications," Sci. Rep., vol. 7, no. 1, pp. 1-11, 2017.

[16] D.-K. Lee et al., "Highly sensitive and selective sugar detection by terahertz nano-antennas," Sci. Rep., vol. 5, no. 1, pp. 1-7, 2015.

[17] L. Razzari et al., "Terahertz dipole nanoantenna arrays: Resonance characteristics," Plasmonics, vol. 8, no. 1, pp. 133-138, 2013.

[18] D. Gray, J. W. Lu, and D. V. Thiel, "Electronically steerable Yagi-Uda microstrip patch antenna array," IEEE Trans. Antennas Propag., vol. 46 no. 5 , pp. 605-608, May 1998 .

[19] C. Feuillet-Palma, Y. Todorov, A. Vasanelli, and C. Sirtori, "Strong near field enhancement in THz nano-antenna arrays," Sci. Rep., vol. 3, no. 1, pp. $1-8,2013$

[20] K. Sengupta and A. Hajimiri, “A $0.28 \mathrm{THz}$ power-generation and beamsteering array in CMOS based on distributed active radiators," IEEE $J$ Solid-State Circuits, vol. 47, no. 12, pp. 3013-3031, Dec. 2012.

[21] F. Caster II et al., "Design and analysis of a W-band 9-element imaging array receiver using spatial-overlapping super-pixels in silicon," IEEE J. Solid-State Circuits, vol. 49, no. 6, pp. 1317-1332, Jun. 2014.

[22] E. R. Brown, A. W. M. Lee, B. S. Navi, and J. E. Bjarnason, "Characterization of a planar self-complementary square-spiral antenna in the $\mathrm{THz}$ region," Microw. Opt. Technol. Lett., vol. 48, no. 3, pp. 524-529, 2006

[23] D. F. Filipovic, S. S. Gearhart, and G. M. Rebeiz, "Double-slot antennas on extended hemispherical and elliptical silicon dielectric lenses,' IEEE Trans. Microw. Theory Techn., vol. 41, no. 10, pp. 1738-1749, Oct. 1993.

[24] G. M. Rebeiz, D. P. Kasilingam, Y. Guo, P. A. Stimson, and D. B. Rutledge, "Monolithic millimeter-wave two-dimensional horn imaging arrays," IEEE Trans. Antennas Propag., vol. 38, no. 9, pp. 1473-1482, Sep. 1990
[25] R. Hincapie and J. E. Sierra, Advanced Transmission Techniques in WiMAX. Norderstedt, Germany: BoD-Books on Demand, 2012.

[26] D. Warmowska, A. K. L. Enrique, A. G. Muñoz, and Z. Raida, "High-gain, circularly-polarized $\mathrm{THz}$ antenna with proper modeling of structures with thin metallic walls," IEEE Access, vol. 8, pp. 125223-125233, 2020.

[27] T. Sharma, G. Varshney, R. S. Yaduvanshi, and M. Vashishath, "Obtaining the tunable band-notch in ultrawideband $\mathrm{THz}$ antenna using graphene nanoribbons," Opt. Eng., vol. 59, no. 4, 2020, Art. no. 047103.

[28] J. Pérez-Urquizo et al., "Monolithic patch-antenna THz lasers with extremely low beam divergence and polarization control," ACS Photon., vol. 8, no. 2, pp. 412-417, 2021.

[29] R. Han et al., "A. 280-GHz Schottky diode detector in 130-nm digital CMOS," IEEE J. Solid-State Circuits, vol. 46, no. 11, pp. 2602-2612, Nov. 2011.

[30] D. Hou et al., "D-band on-chip higher-order-mode dielectric-resonator antennas FED by half-mode cavity in CMOS technology," IEEE Antennas Propag. Mag., vol. 56, no. 3, pp. 80-89, Jun. 2014.

[31] J. Grzyb, Y. Zhao, and U. R. Pfeiffer, “A288-GHz lens-integrated balanced triple-push source in a 65-nm CMOS technology," IEEE J. Solid-State Circuits, vol. 48, no. 7, pp. 1751-1761, Jul. 2013.

[32] X.-D. Deng, Y. Li, C. Liu, W. Wu, and Y.-Z. Xiong, "340 GHz on-chip 3-d antenna with 10 DBi gain and 80\% radiation efficiency," IEEE Trans. Terahertz Sci. Technol., vol. 5, no. 4, pp. 619-627, Jul. 2015.

[33] S.-Y. Suh, W. L Stutzman, and W. A Davis, "A new ultrawideband printed monopole antenna: The planar inverted cone antenna (PICA)," IEEE Trans. Antennas Propag., vol. 52, no. 5, pp. 1361-1364, May 2004

[34] S.-Y. Suh, W. Stutzman, W. Davis, A. Waltho, and J. Schiffer, "A novel CPW-fed disc antenna," in Proc. IEEE Antennas Propag. Soc. Symp., 2004, vol. 3, pp. 2919-2922

[35] S.-Y. Suh, "A Comprehensive investigation of new planar wideband antennas," Ph.D. dissertation, Elect. Comput. Eng. Dept., Virginia Polytech. Inst. State Univ., Blacksburg, VA, USA, Jul. 2002.

[36] A. Abohmra et al., "Terahertz antenna array based on a hybrid Perovskite structure," IEEE Open J. Antennas Propag., vol. 1, pp. 464-471, 2020.

[37] A. R. Conn, Nicholas I. M. Gould, and P. L. Toint, Trust-Region Methods (MPS-SIAM series on optimization). Philadelphia, PA, USA: SIAM, 2000.

[38] J. R. James, P. S. Hall, and C. Wood, Microstrip Antenna: Theory and Design, (IEE Electromagnetic Waves Series 12). London, U.K.: IET, 1986.

[39] M. Walther, D. G. Cooke, C. Sherstan, M. Hajar, M. R. Freeman, and F. A. Hegmann, "Terahertz conductivity of thin gold films at the metal-insulator percolation transition," Phys. Rev. B, vol. 76, no. 12 2007, Art. no. 125408

[40] A. V. Kabashin et al., "Nanofabrication with pulsed lasers," Nanoscale Res. Lett., vol. 5, no. 3, pp. 454-463, 2010.

[41] P. Haring et al., "Measurement of the dielectric constant and loss tangent of high dielectric-constant materials at terahertz frequencies," IEEE Trans. Microw. Theory Techn., vol. 51, no. 4, pp. 1062-1066, Apr. 2003. 